\title{
Flight Demonstration of Provably Safe Closely Spaced Parallel Approaches
}

\author{
Rodney Teo*, Jung Soon Jang\# ${ }^{\#}$ and Claire J. Tomlin ${ }^{\dagger}$ \\ Stanford University, CA 94305, USA \\ e-mail: * rodney.teo@stanfordalumni.org, \# jsjang@stanford.edu, \\ $\dagger$ tomlin@stanford.edu
}

\begin{abstract}
In poor visibility, paired approaches to airports with closely spaced parallel runways are not permitted, thus halving the arrival rate. With Global Positioning System technology, datalinks and cockpit displays, this could be averted. One important problem is ensuring safety during a blundered approach by one aircraft. This is on-going research. A danger zone around the blunderer is required. If the correct danger zone could be calculated, then it would be possible to get $100 \%$ of clear-day capacity in poor-visibility days even on 750 foot runways. The danger zones vary significantly (during an approach) and calculating them in real time would be very significant. Approximations (e.g. outer bounds) are not good enough. This paper presents a way to calculate these danger zones in real time for a very broad class of blunder trajectories.

The approach in this paper differs from others in that it guarantees safety for any possible blunder trajectory as long as the speeds and turn rates of the blunder are within certain bounds. In addition, the approach considers all emergency evasive maneuvers whose speeds and turn rates are within certain bounds about a nominal emergency evasive maneuver. For all combinations of these blunder and evasive maneuver trajectories, it guarantees that the evasive maneuver is safe. For more than 1 million simulation runs, the algorithm shows a $\mathbf{1 0 0 \%}$ rate of Successful Alerts and a $0 \%$ rate of Collisions Given an Alert.

The evaluation of the proposed scheme was demonstrated with two different types of test platforms, the Stanford DragonFly unmanned aerial vehicles and the Boeing testbed of F-15 and T-33 aircraft. The experiment involved two aircraft, where one aircraft is assigned as pursuer and the other as evader. The algorithm proved the guaranteed safety (maintaining a minimum separation distance) for every single flight test performed, and demonstrated that it can run it in real-time.

Keywords: unmanned aerial vehicle, collision avoidance, differential game theory, optimal control theory.
\end{abstract}

\section{Introduction}

$\mathrm{C}$ losely spaced parallel approaches (CSPA) refer to the simultaneous approaches of pairs of aircraft to $\checkmark$ airports with parallel runways that are closely spaced. There are two types of parallel approaches: independent and dependent. Independent approaches refer to approaches of pairs of aircraft where there is no restriction in the longitudinal spacing between them. Dependent approaches, on the other hand, impose a restriction on the longitudinal separation between the aircraft pair: Neither aircraft is permitted to violate the minimum longitudinal separation.

CSPA can be conducted only in Visual Meteorological Conditions (VMC), i.e., when there is clear visibility. Thus, in IMC, Instrument Meteorological Conditions (IMC), i.e., when visibility is poor, the airport arrival rate is reduced by half. This also has an upstream effect all the way to the airports where aircraft destined for the affected airports originate. The departures of these aircraft are delayed because the destination airports have to slow down the rate at which they accept aircraft for landing.

Two factors affect the safety of a CSPA operation. They are blunders and wake vortices. A blunder refers to some trajectory that deviates from the normal approach path that could be a result of human error, 


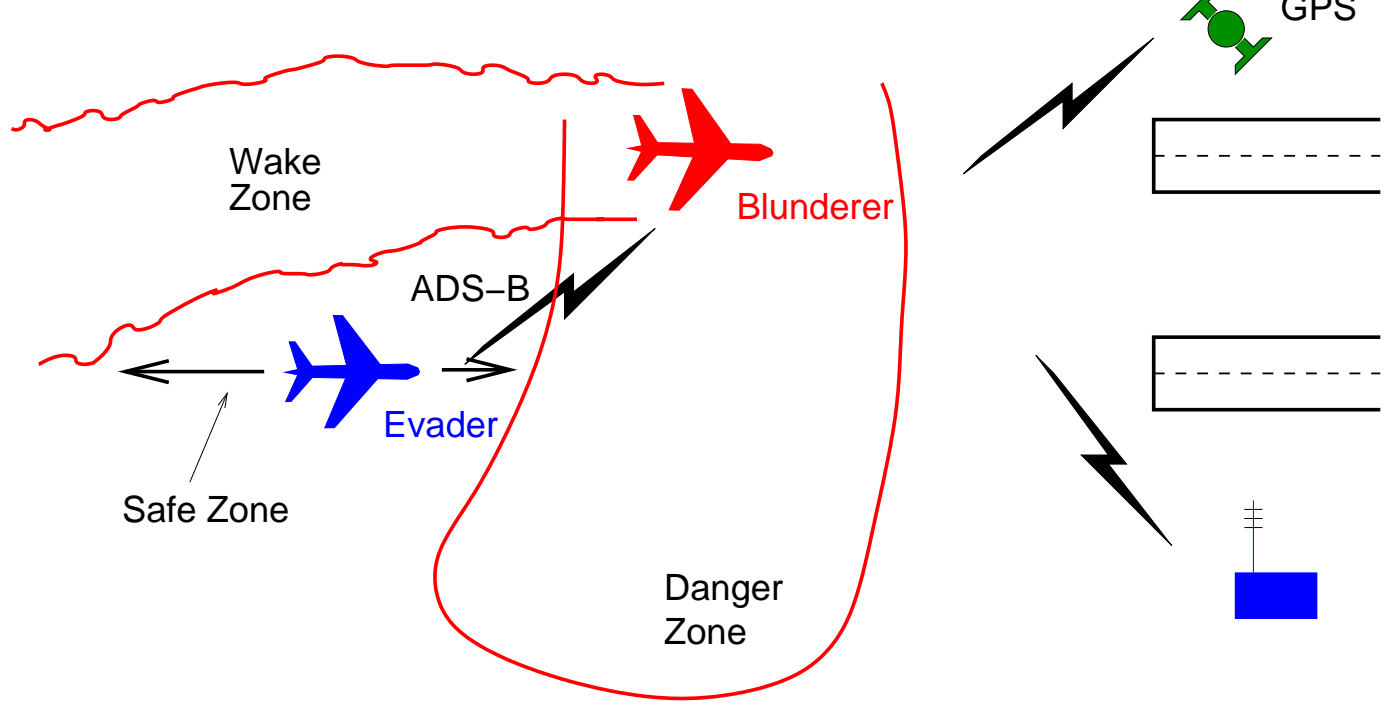

Figure 1. Danger and Wake Vortex Zones in CSPA.

instrument or mechanical failure, or strong gusts; while wake vortices refer to the strong and 'turbulent' air currents that are left in the trail of an aircraft. The regions of effect of blunders and wake vortices can be depicted as two zones (see Figure (1). The zone due to blunder is referred to as the danger zone, as in the literature, ${ }^{1}$ while the zone due to wake vortex is referred to as the wake zone. In this figure, only the danger and wake zones posed by the lead aircraft to the trailing aircraft are shown. Similarly, there are two such zones of the trailing aircraft imposed on the lead aircraft. These two zones impose a forward and rearward limit for following the adjacent aircraft. The zone in between the limits is thus the safe zone.

GPS navigation using the Wide Area Augmentation System ${ }^{2}$ or Local Area Augmentation System, coupled with the Automatic Dependent Surveillance (Broadcast) (ADS(B)), ${ }^{3}$ which is a wireless datalink among aircraft, as well as cockpit displays, could well enable CSPA in IMC if the right procedures and algorithms are developed. There is considerable research focused on enabling such operations. They could be broadly categorized under system design and research, ${ }^{4-18}$ alerting algorithms research, ${ }^{19-22}$ procedure and humanmachine interface research, ${ }^{1,17,22-25}$ and wake-related research. ${ }^{26-32}$

The focus of this paper is the flight demonstration of a new CSPA algorithm for ensuring safety against blunders. It is a summary of the key results presented in Ref. 33. This new CSPA algorithm has been presented in Ref. 34 and 33. Earlier versions are in Ref. 35 and 36. Other previously proposed algorithms are the Honeywell AILS Segmented Alerting algorithm, ${ }^{19,20}$ the algorithm by Landry and Pritchett, ${ }^{22}$ and the Massachusetts Institute of Technology (MIT) Probabilistic Alerting Algorithm. ${ }^{21}$ These algorithms, however, all assume some specific set of blunder trajectories. These algorithms can thus guarantee safety only for the specific set of blunder trajectories assumed. Consequently, a loss of separation could occur for blunder trajectories that are not assumed in these algorithms. The algorithm demonstrated in this paper does not have that constraint.

The new algorithm demonstrated in this paper is based on ideas from differential game theory. It considers all blunder trajectories that are possible as long as their speeds and turn rates are within certain bounds. In addition, it considers all emergency evasive maneuvers whose speeds and turn rates are within certain bounds of the nominal emergency evasive maneuver. And, for all combinations of these blunder and evasive maneuver trajectories, the approach guarantees that the evasive maneuver is safe. This is the key difference in effectiveness in the new approach of this algorithm compared to the other work. The resulting computation provides a danger zone. This computation is also made to run in real-time. This is also very significant as it enables the computation of the correct danger zone at each time step with the correct states. Approximations of the danger zone with worst-case states are not good enough as they would be overly conservative, thus requiring very large and therefore impractical longitudinal separation distances between aircraft during the parallel approach. The ability to calculate the correct danger zones in real time can enable closely spaced parallel approaches in poor visibility at the same level as in clear day conditions. 


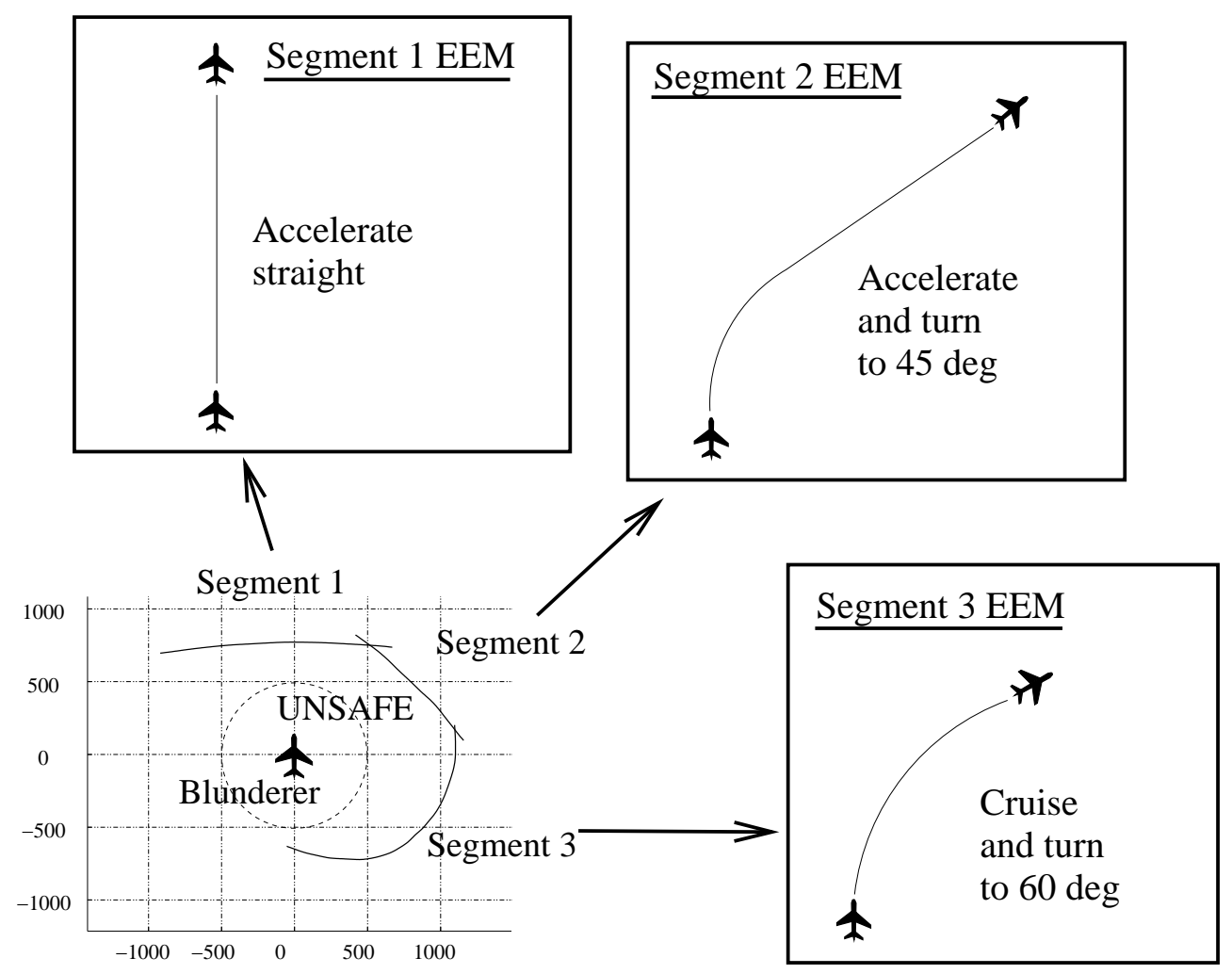

Figure 2. A sample danger zone result generated using 3 different EEMs.

The key contribution presented in this paper is to demonstrate the implementation of the new algorithm through an autonomous closely spaced parallel approach by both aircraft with one of them programmed to blunder. The other aircraft is programmed to run the algorithm on board using its own information and the information transmitted from the blundering aircraft. It responds according to the result of the algorithm and conducts an autonomous evasive maneuver when required. This flight demonstration was successfully conducted and it shows the implementation of the algorithm, in particular, demonstrating that it can be run in real-time. This is the focus of this paper.

The key result of the above contributions is as follows: With the ability to calculate the correct danger zones in real time for a very broad class of blunder trajectories, and with the necessary sensors and datalink, and the appropriate procedures in place, it would be possible to get $100 \%$ of clear-day capacity in poorvisibility days even on 750 foot runways.

The paper is organized as follows: Section II briefly describes the new CSPA algorithm, Section III, its implementation considerations and its validation results. Section IV describes the flight demonstration of the new algorithm. Section $\mathrm{V}$ concludes the paper.

\section{Brief Description of the CSPA Algorithm}

This section provides a brief description of the new CSPA algorithm. The full details can be found in Ref. 34 .

As with previous research, the central assumption is made that state information (location and velocity) of each aircraft is available from either a Local Area Augmentation DGPS system (LAAS) ${ }^{20}$ or a Wide Area Augmentation DGPS system (WAAS) ${ }^{16}$ and transmitted to the other aircraft through an Automatic Dependent Surveillance-Broadcast (ADS-B) ${ }^{3}$ datalink.

Essentially, a useful way to see the value of the new algorithm is in terms of the resulting danger zones it forecasts and avoids. An example of a danger zone is shown as the "UNSAFE" region in Figure 2, Segments 1 to 3 are obtained using the Emergence Escape Maneuvers (EEMs) labeled 1 to 3 respectively, in Figure 2. These EEMs are also shown in the figure. The danger zone is a region in which an EEM that is initiated 


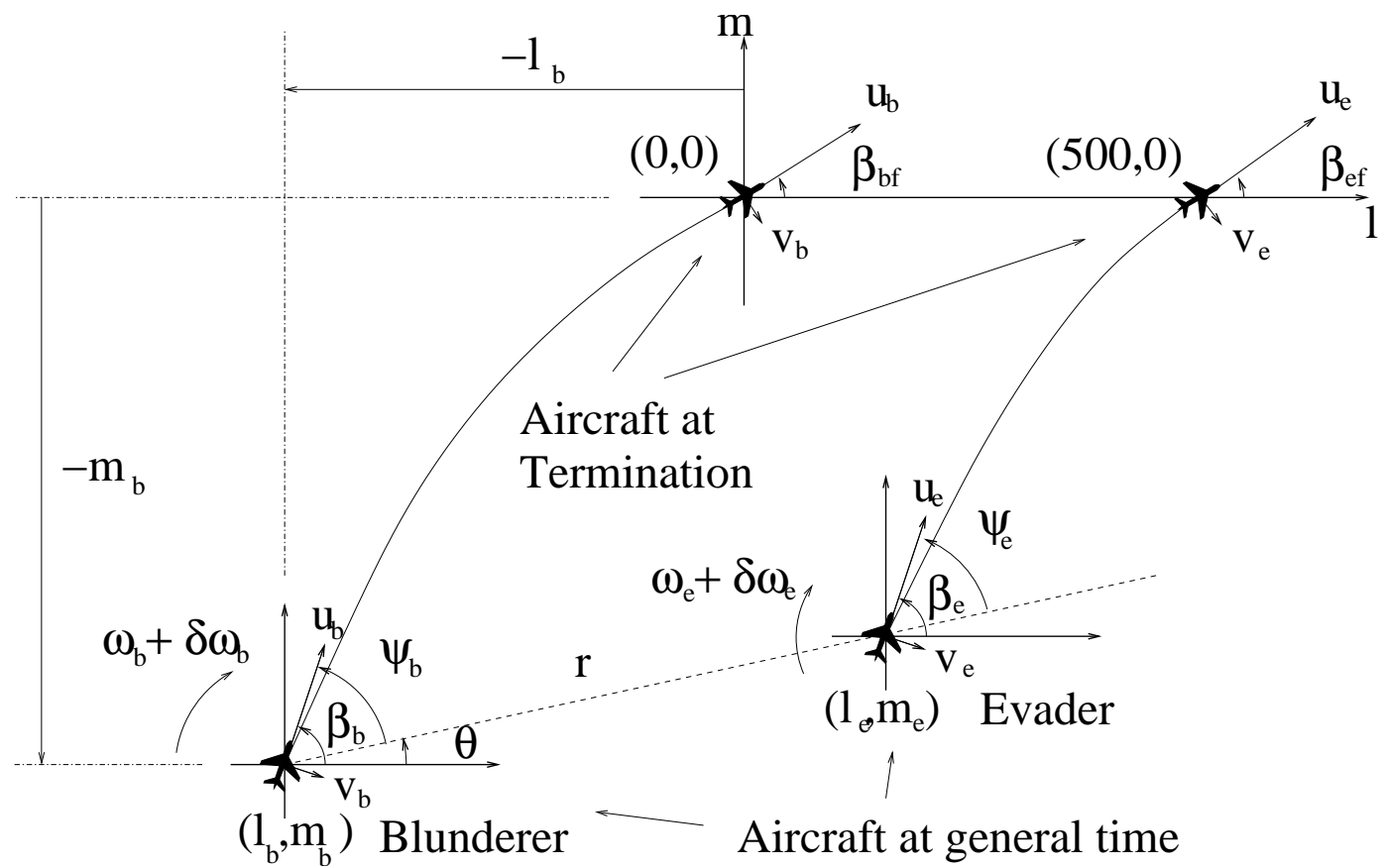

Figure 3. Reference Frame.

inside it is not guaranteed to be safe. An EEM initiated outside or on the boundary of the danger zone, on the other hand, is guaranteed to be safe provided that the initiated EEM is the appropriate one. The appropriate EEM is the one that is used to generate the boundary that is being encroached. For example, if the evader is encroached by Segment 2 of the boundary, then the appropriate EEM to conduct is the Segment 2 EEM. In fact, so long as the Segment 2 EEM is conducted outside Segment 2 of the boundary, that EEM is guaranteed to be safe.

Two EEMs are used for dependent approaches. They correspond with the Segment 2 EEM which is an accelerate, climb and turn to 45 deg EEM, and with the Segment 3 EEM which is a maintain speed, climb and turn to 60 deg EEM. The Segment 1 EEM is an accelerate and climb straight EEM. This EEM is not used in the algorithm validation nor in the flight demonstration of the algorithm. It is not meant to be a part of the algorithm. It is however presented in the results to illustrate its benefit compared with the Segment 2 EEM in some cases.

The danger zone is of course not static. It is computed in real-time and its size and shape is a function of the aircraft speeds, relative heading, and worst-case bounds on the variation of their speeds and turn rates from the nominal. Thus, the danger zone will change in shape and size during a CSPA.

The key characteristic of the new algorithm is that it guarantees safety. To give a more precise definition of this statement, consider the reference frame as shown in Figure 3, The origin of the reference frame coincides with the blunderer position at termination (which is the closest point of approach) and the $l$-axis passes through the evader position at termination. The location of the aircraft with respect to the reference frame is given by $l_{i}$ and $m_{i}$ where the subscript $i$ represents the aircraft; $b$ for blunderer and $e$ for evader. $\beta_{i}$ refers to the heading of aircraft $i$ as measured from the $l$-axis in a counter-clockwise direction.

For each aircraft, the actual motion resulting from the throttle, elevator, aileron and rudder inputs is taken to be $u_{i}, v_{i}$ and $\delta \omega_{i}$. They are, respectively, the controlled changes in forward speed, lateral speed and the variations in turn rate about the nominal turn rate of aircraft $i$. These will be called herein "control inputs". In a sense, they are the control inputs of the system which represents the kinematic motion of each aircraft.

The 2-dimensional kinematic equations of motion for the blunderer aircraft are given as:

$$
\begin{aligned}
& \dot{l}_{b}=u_{b} \cos \left(\beta_{b}\right)+v_{b} \sin \left(\beta_{b}\right) \\
& \dot{m}_{b}=u_{b} \sin \left(\beta_{b}\right)-v_{b} \cos \left(\beta_{b}\right) \\
& \dot{\beta}_{b}=-\delta \omega_{b}
\end{aligned}
$$

$$
4 \text { of } 15
$$


The bounds on the control inputs (of the kinematic motion representation of each aircraft) are given as:

$$
\begin{aligned}
u_{b}^{\min } & \leq u_{b} \leq u_{b}^{\max } \\
-v_{b}^{\max } & \leq v_{b} \leq v_{b}^{\max } \\
-\delta \omega_{b}^{\max } & \leq \delta \omega_{b} \leq \delta \omega_{b}^{\max }
\end{aligned}
$$

The equations of motion for the evading aircraft are:

$$
\begin{aligned}
& \dot{u}_{e}=a_{e} \\
& \dot{l}_{e}=u_{e} \cos \left(\beta_{e}\right)+v_{e} \sin \left(\beta_{e}\right) \\
& \dot{m}_{e}=u_{e} \sin \left(\beta_{e}\right)-v_{e} \cos \left(\beta_{e}\right) \\
& \dot{\beta}_{e}=-\left(\omega_{e}+\delta \omega_{e}\right)
\end{aligned}
$$

The bounds on the control inputs (of the kinematic motion representation of the evader aircraft) for the Segment 1 and 2 EEMs are given as:

$$
\begin{aligned}
a_{e}^{\min } & \leq a_{e} \leq a_{e}^{\max } \\
-v_{e}^{\max } & \leq v_{e} \leq v_{e}^{\max } \\
-\delta \omega_{e}^{\max } & \leq \delta \omega_{e} \leq \delta \omega_{e}^{\max }
\end{aligned}
$$

For the Segment $3 \mathrm{EEM}, a_{e}=0$ and the bounds on $u_{e}$ are:

$$
u_{e}^{\min } \leq u_{e} \leq u_{e}^{\max }
$$

Note that $v_{i}$ represents the lateral speed component. In this representation, the control inputs can instantaneously take on any value within these bounds. Instantaneous switches will of course not occur in actual aircraft speeds and turn rate. This assumption, though, will make the analysis conservative and, more importantly, it will capture all the possible aircraft maneuvers. For the blunderer, $\omega_{b}=0$ and $\delta \omega_{b}$ represents the variation in turn rate which is bounded equally in both the left and right directions. For the evader, $\omega_{e}$ is the nominal turn rate in the evasive maneuver and $\delta \omega_{e}$ represents the variation in the turn rate from the nominal. The evader is assumed to be initially in level flight during the CSPA. When an EEM is conducted, there will be a time lag for the evader to achieve the bank angle for the EEM. A delay in achieving the nominal turn rate is thus included to account for this. For the delay period, the evader is assumed to have zero turn rate. In this way, the time lag for banking is approximated.

Given the above motion representation of the blunderer and the evader, safety guarantee implies that for all blunder trajectories and all evader trajectories about the respective the EEM, the separation distance between the two aircraft will never violate the threshold minimum (which is chosen to be $500 \mathrm{ft}$ in this paper). For cases when the evader is less maneuverable than the blunderer (for e.g., when $\delta \omega_{b}>>\omega_{e}$ ), there will exist blunderer trajectories that can violate the threshold. In these cases, safety is guaranteed for the duration required by the evader to complete the EEM (for e.g., in the Segment 3 case, EEM completion occurs when the evader has turned to $60 \mathrm{deg}$ ).

It is also worth noting that the EEM is open-loop safe in that no state information of the blunderer is needed during the EEM. The EEM is guaranteed to be safe regardless of the trajectory of the blunderer so long as its speeds and turn rate are within the bounds used to compute the danger zone that initiated the EEM. Thus, no information of the blunderer is required during the EEM to ensure safety as long as the blunder's actual trajectory obeys the bounds in its speeds and turn rate.

These danger zones can also be used to determine the minimum safe runway separation for independent approaches. For dependent approaches, the danger zone can be used to determine the minimum longitudinal separation (MLS) between aircraft pairs. During normal operations, the aircraft will stay above these minimums and the danger zone will be computed on-line to alert the pilot or autopilot when to carry out the appropriate EEM in the event of blunders.

In this paper, the results presented are for an evader that is performing the approach on the right of the blunderer. Thus, the turns in the EEMs above are turns to the right (away from the blunderer). The results for an evader performing the approach on the left is simply a mirror image of the results shown here. 


\section{Implementation and Validation}

The schematic for the implementation of the new algorithm is shown in Figure 4. The algorithm runs on the 'own aircraft'. It takes speed and heading information from both aircraft and computes the danger zone.

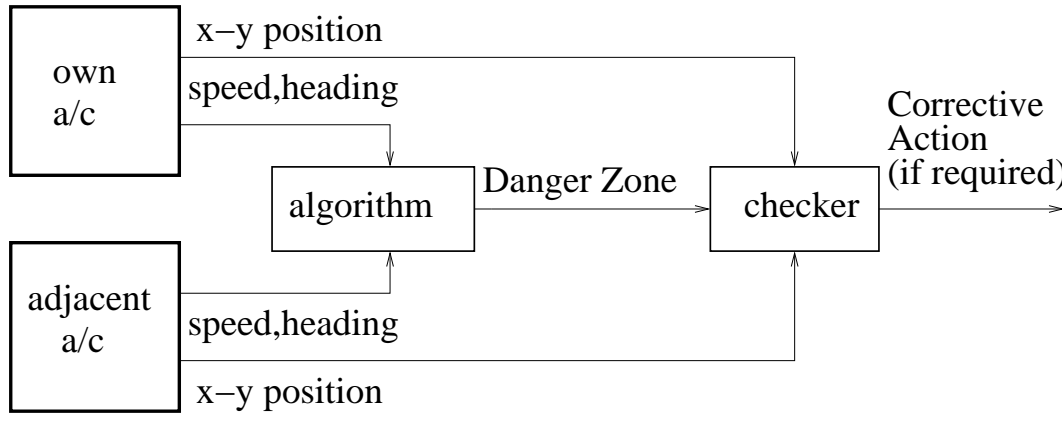

Figure 4. Schematic showing Algorithm Implementation.

With the position information from both aircraft, it then performs the check of whether the boundary of the danger zone encroaches the position of the own aircraft. If so, it activates the necessary EEM. During the EEM, speed and heading information of the blundering aircraft will not be required unless they change beyond the limits expected.

Some issues and considerations regarding the implementation of the algorithm are as follows.

- Communication dropouts: It is reasonable to expect communication dropouts over a wireless link. The algorithm takes this into account and guarantees safety despite dropouts.

- Simplification: As it is, the danger zone boundary, for the most part, is given in the form of a series of parameterized explicit analytical expressions (only one case involves a bisection search algorithm). To simplify the computation further, a slightly conservative approximation by a polygon is used, as shown in Figure 5 .

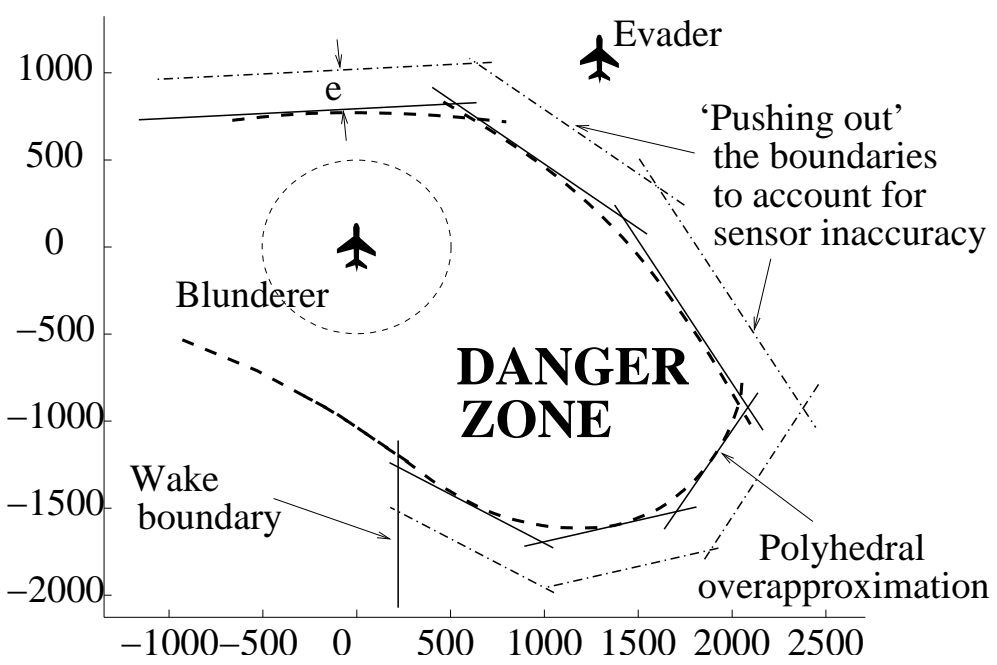

Figure 5. Conservative Approximation of Danger Zone for On-line Computation.

Using Microsoft Visual C++ ver 6.0 on a Dell Pentium 3 machine, each computation of this approximation of the danger zone can run in $0.05 \mathrm{sec}$.

- Sensor inaccuracy: Sensor inaccuracy is accounted for assuming WAAS-like characteristics. This is achieved by 'pushing out' the sides of the polygon as shown in Figure 5.

- Cockpit display: A cockpit display of the danger zones to the pilot have been developed by Jennings, ${ }^{17}$ as shown in Figure 6 .

The danger zone computation is based on a 2-dimensional kinematic model which is engineered to contain realistic aircraft trajectories. These constitute approximate aircraft models. A validation of the implementation of the algorithm is thus required. Also, it is useful in verifying the algorithm's strengths.

$$
6 \text { of } 15
$$




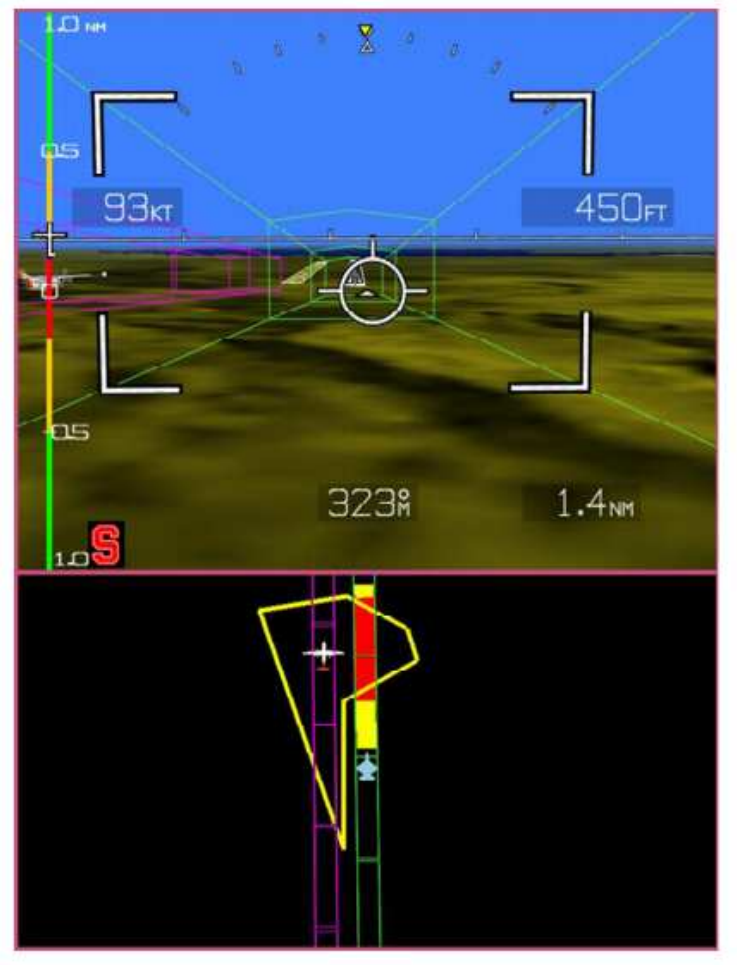

Figure 6. Cockpit Displays (for aircraft on the right) showing a normal approach (Courtesy of Chad W. Jennings $\left.{ }^{17}\right)$.

and bounds on control inputs. The validation approach is in the Honeywell approach, ${ }^{20,37}$ three metrics are used to evaluate the validation results. They are the probabilities of

1. Successful Alerts (SA): this refers to events where alerts are given, the EEMs are executed, and no collisions occur; and if the EEMs were not executed, collisions would have occurred.

2. Unnecessary Alerts (UA): this refers to events where alerts are given, the EEMs are executed, and no collisions occur; and if the EEMs were not executed, no collision would have occurred either. If the alert were given because the other aircraft is blundering, the alert will not be deemed as unnecessary. However, such cases are still categorized as UAs in order to be consistent with the literature. ${ }^{20,37}$

3. Collision, Given an Alert (CGA): this refers to events where alerts are given, the EEMs are executed, and yet, collisions occur; and if the EEMs were not executed, no collisions would have occurred.

The results, together with those of the Honeywell AILS Segmented ${ }^{20}$ and MIT Probabilistic ${ }^{21}$ Alerting Algorithms, and the corresponding formulae for the metrics are given in Table 1, In the table, the acronym CSPA refers to the new algorithm demonstrated in this paper, and is referred to as the CSPA algorithm, while AILS refers to the Honeywell algorithm and MIT refers to the MIT algorithm. To compare the results with the other two algorithms, some background is first given. The Honeywell algorithm is designed for independent approaches with runway spacings of $2500 \mathrm{ft}$ or greater. Thus, only the results for a runway spacing of $2500 \mathrm{ft}$ are available. The thresholds used in the Honeywell algorithm to trigger alerts are obtained empirically through simulating a large of number of parallel approaches with blunders. The thresholds picked are the ones that satisfactorily prevent collision. It is not stated in the Honeywell paper ${ }^{20}$ what 'satisfactory' quantitatively corresponds to. The MIT algorithm, on the other hand, is designed so that the probability of collision in the event of a blunder is 0.001 . This criterion is considered to be the acceptable level of risk in the Precision Runway Monitor System studies. ${ }^{38}$

The CSPA algorithm has 100\% SA and 0\% CGA whereas both the Honeywell and MIT algorithms have some Collisions, Given Alerts (CGAs). With regard to Unnecessary Alerts (UA), the CSPA algorithm 
Table 1. Metrics for three algorithms: \% of runs.

\begin{tabular}{|c|c|c|c|c|c|c|c|}
\hline & & $750 f t$ & \multicolumn{2}{|c|}{$1700 \mathrm{ft}$} & \multicolumn{3}{c|}{$2500 \mathrm{ft}$} \\
\hline \hline & Formula & CSPA & CSPA & MIT & CSPA & AILS & MIT \\
\hline SA & $\frac{C D+F A}{C D+F A+M D+I C+L A}$ & $100 \%$ & $100 \%$ & $99.5 \%$ & $100 \%$ & $99.9 \%$ & $99.3 \%$ \\
\hline UA & $\frac{I C+F A}{C D+F A+M D+I C+L A}$ & $98.78 \%$ & $77.14 \%$ & $55.5 \%$ & $69.02 \%$ & $93.7 \%$ & $48.7 \%$ \\
\hline CGA & $\frac{I C+L A}{C D+F A+I C+L A}$ & $0 \%$ & $0 \%$ & $0.5 \%$ & $0 \%$ & $0.1 \%$ & $0.7 \%$ \\
\hline
\end{tabular}

has a lower UA rate than the Honeywell algorithm but a higher one than the MIT algorithm. This latter comparison may appear to be a disadvantage. However, as explained earlier, alerts in the UA category would be considered necessary when they are raised by blunders that have the potential to later cause a collision should their trajectories be modified mid-course. This viewpoint is also expressed by Honeywell in their paper. $^{20}$ The blunders that were correctly rejected (CR) by the CSPA algorithm are those that did not have the potential as yet to cause a collision. In CSPA, it is more important to ensure that False Alarms (FAs) do not occur during normal aircraft approaches with normal flight technical error (FTE). With the CSPA algorithm, no False Alarms will be raised so long as the aircraft maintain their longitudinal separation according to the MLS, as explained in Section II.

The MIT algorithm might have produced better SA and CGA results if it were designed for a lower collision-probability value. However, it must be noted that it still relies on a specified blunder trajectory set, whereas the CSPA algorithm does not.

In simulation, the new algorithm has been successfully demonstrated to work according to expectations and is able to guarantee safety as long as the blunder trajectories are captured by the model and are within the bounds on the control inputs assumed.

\section{Flight Demonstration}

This section describes the flight demonstration of the implementation of the danger zone computation algorithm on the Stanford DragonFly UAVs and the Boeing testbed of F-15 and T33 aircraft and discuss the results.

\section{A. Dual UAV Demonstration}

In this flight demonstrations, DF2 is designated the Own Ship which will run the CSPA algorithm and conduct the evasive maneuver when required. DF3 is designated the Other Ship which will blunder in its 'final approach'. Segment 1 of the Danger Zone is not used. Segment 2 is used in its place. In the demonstration, both aircraft will conduct the closely spaced parallel approach autonomously. DF3 will be programmed to blunder in its approach at a certain point. The algorithm will run on board DF2 and will trigger an EEM according to the danger zone computation. When triggered to conduct the EEM, DF2 will fly the EEM autonomously.

Prior to the flight demonstration, a ground taxiing demonstration is conducted. For the ground taxiing demonstration, ground taxiing controllers had to be designed. In addition, a navigation algorithm which blends GPS measurements with IMU measurements through an Extended Kalman Filter is designed. The ground taxiing phase is conducted prior to the in-flight phase so that valuable experience in conducting the demonstration is gained without risking the UAVs in the flight phase. The benefits of the ground taxiing phase are as follows:

- Testing Software Readiness. The ground taxiing phase tests that the CSPA algorithm, the server and the client processes can communicate with each other and run simultaneously at the desired rates. It also shows that the CSPA algorithm has been coded correctly to generate the results that match the Matlab version of the algorithm.

- Partially Testing System Readiness. This phase also partially checks that the dual UAVs are ready for operations. This includes checking that the necessary logistics are available for engine starting, battery 


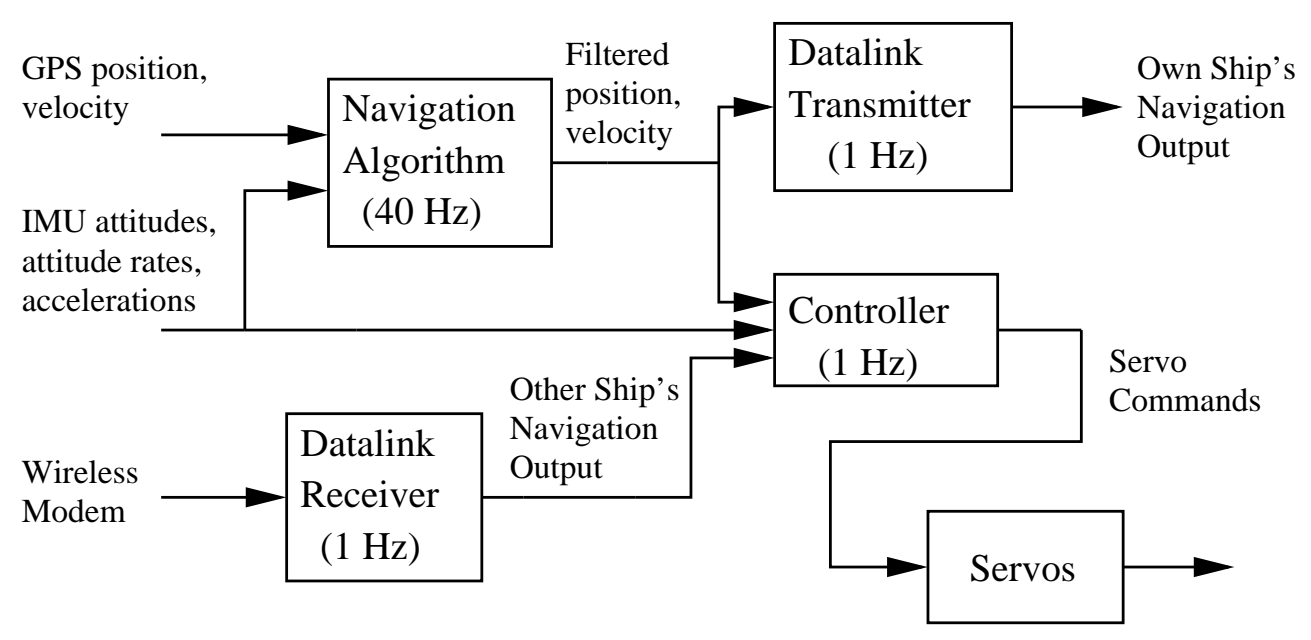

Figure 7. Top Level Architecture of each UAV.

charging, refuelling, etc, that the UAVs can be switched between manual and flight computer control, that the installed sensors work, and that the procedures for operations run smoothly.

After the ground taxiing demonstration, parameter identification of the flight dynamics of the aircraft are obtained experimentally and the flight controllers for airspeed hold, heading hold, bank angle hold, altitude hold, and straight line tracking are designed and flight tested. Thereafter, the flight demonstration could commence. For the demonstrations, each UAV has a top level architecture that is shown in Figure 7, Below, the ground taxiing results are first presented followed by the flight results.

\section{Ground Taxiing Demonstration}

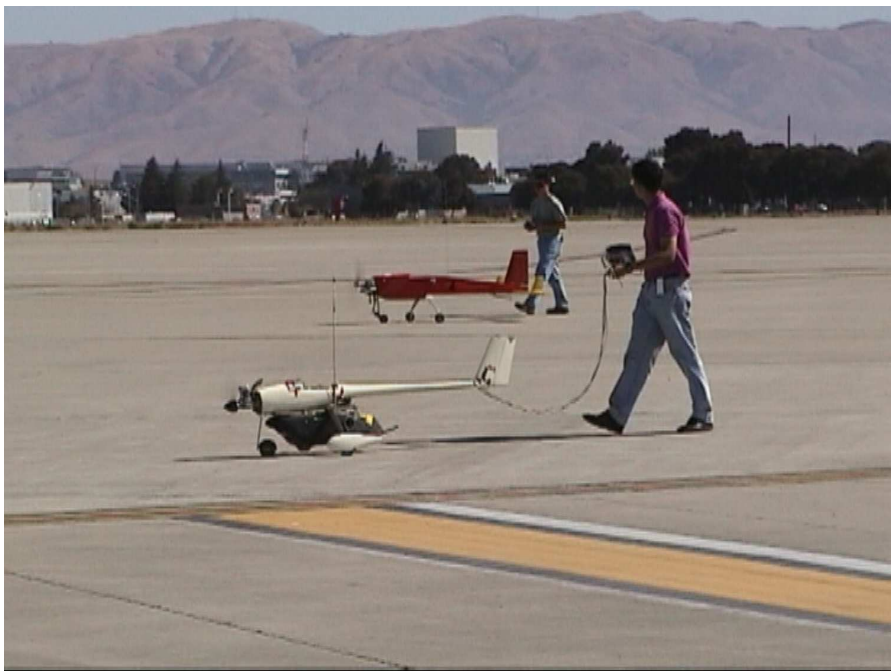

Figure 8. Ground Taxiing Demonstration.

Figure 8 shows the demonstration conducted at Moffett Federal Airfield. About 10 runs are made, each with a different relative start position. In all of the runs, the distance between the two UAVs at the closest point of approach is greater than the threshold of $8 \mathrm{~m}$. The results of two runs are shown in Figures 9 and 10. In Figure 9, the EEM turns out to be the one associated with Segment 2 of the danger zone, i.e., acceleration and turn to 45 deg. In Figure 10, the EEM is the one associated with Segment 3 of the danger zone, i.e., coast and turn to 60 deg. For the former case, the separation distance between the two UAVs almost exceeded the threshold. This is because DF3 turned at almost $10 \mathrm{deg} / \mathrm{s}$ in the experiment whereas a lower value of $5 \mathrm{deg} / \mathrm{s}$ is assumed to be its maximum turn rate in computing the danger zone. In the latter case, the separation distance between the two UAVs is above the threshold.

The completion of the ground demonstration shows that the CSPA algorithm can run on this dual UAV test bed in real-time. 

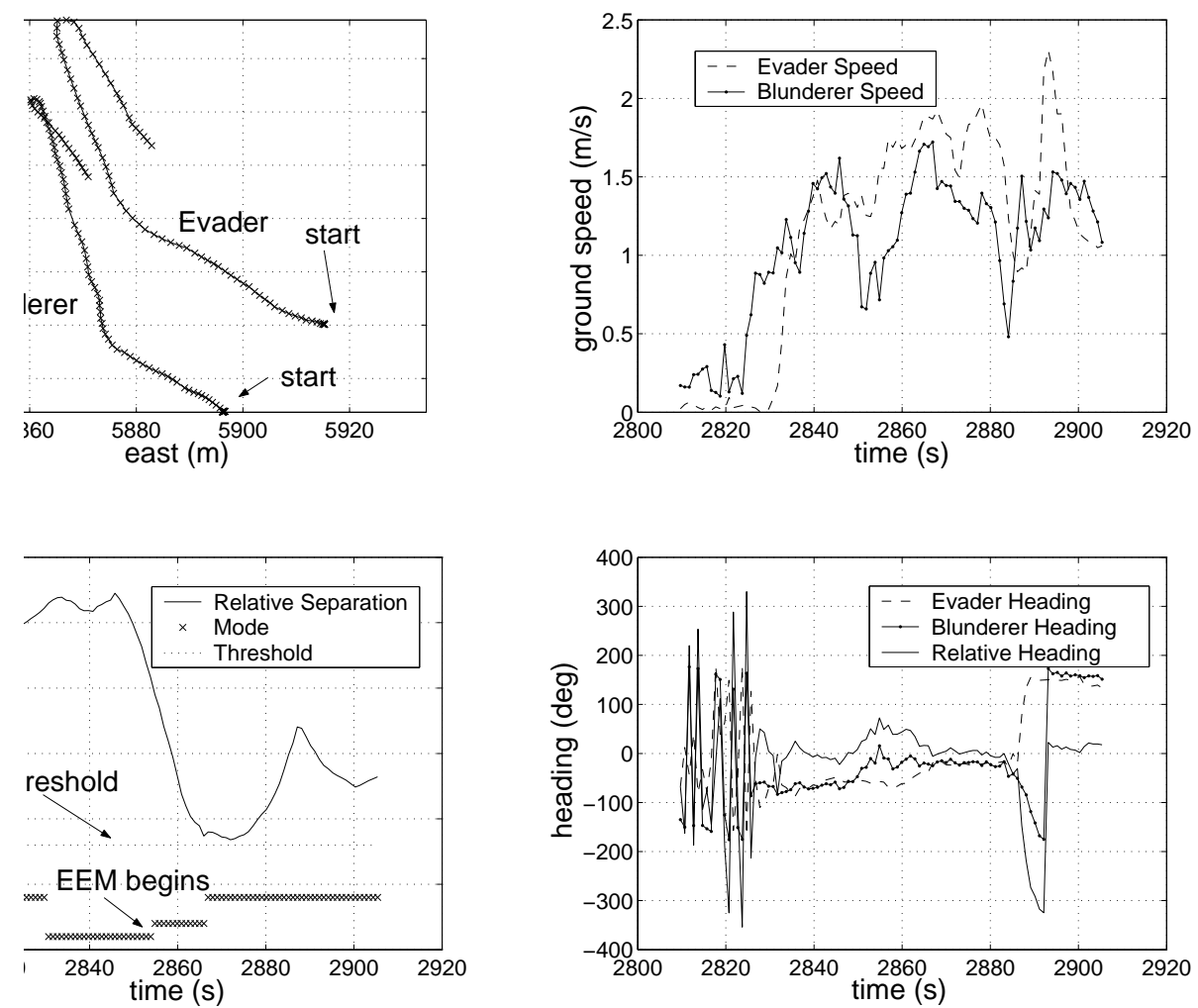

Figure 9. Ground Taxiing Demonstration: Accelerate, Turn 45 deg EEM.
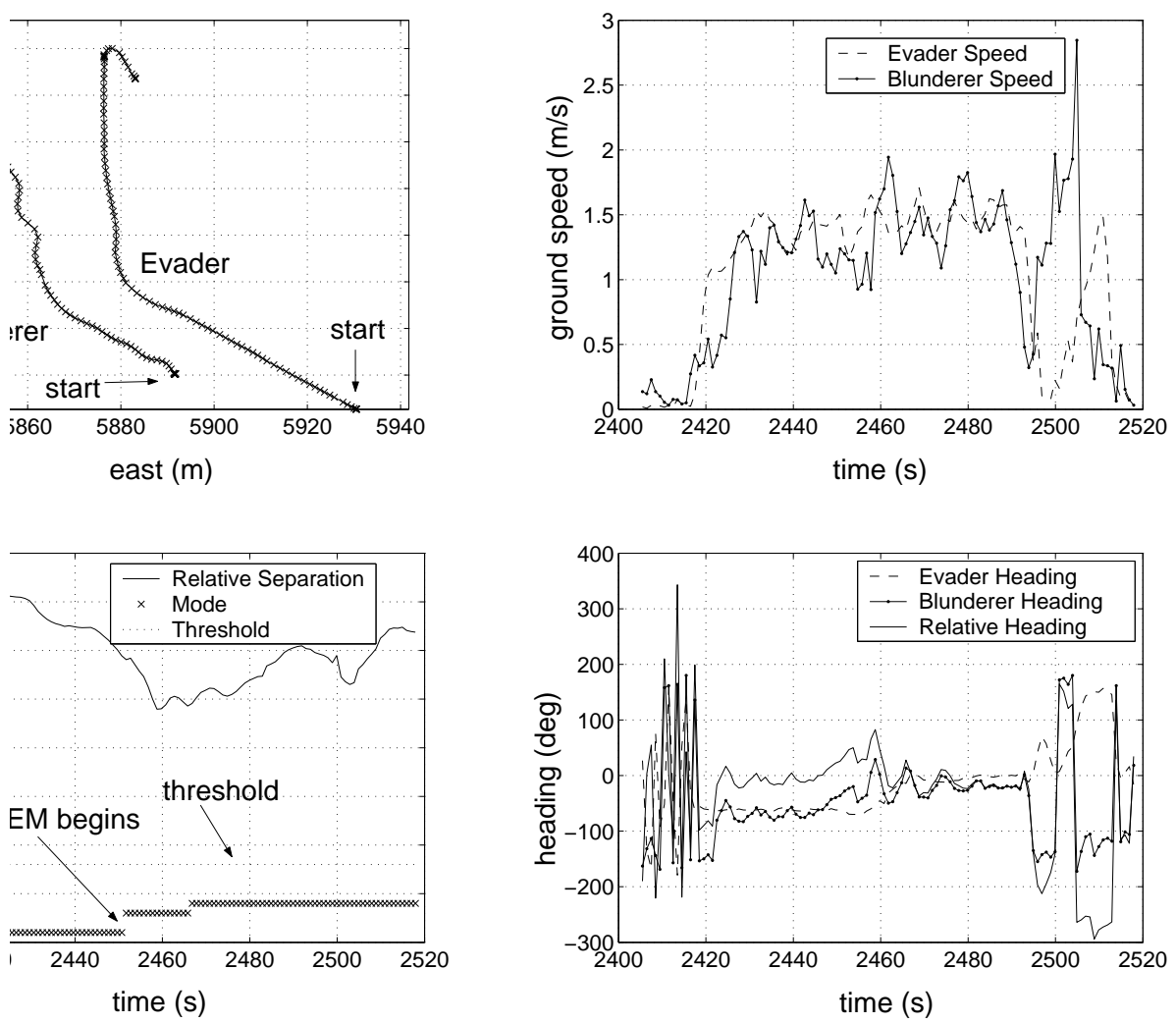

Figure 10. Ground Taxiing Demonstration: Coast, Turn 60 deg EEM. 


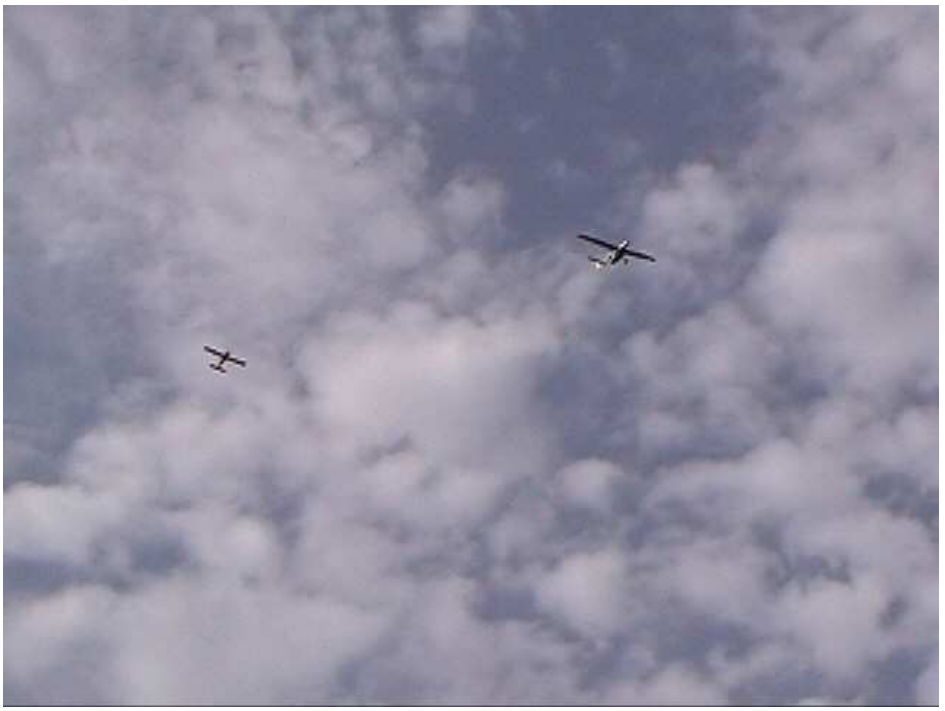

Figure 11. In-Flight Demonstration.
To reiterate, the purpose of the in-flight demonstration is to show that the algorithm can be implemented and in particular, to demonstrate that it can run in real-time.

Figure 11 shows the demonstration conducted over Moffett Federal Airfield. Over twenty runs are made with DF3 blundering and DF2 performing the EEM as triggered by the danger zone computation algorithm. All runs are successful. Sample results are shown in Figure 12. The former emulates an independent approach scenario where there is no restriction in the longitudinal spacing between the two aircraft during normal approaches. The latter emulates a dependent approach scenario.

Note that the separation threshold is not violated. The flight demonstration shows the implementation of the algo-

rithm and in particular, demonstrates that it can run in real-time.

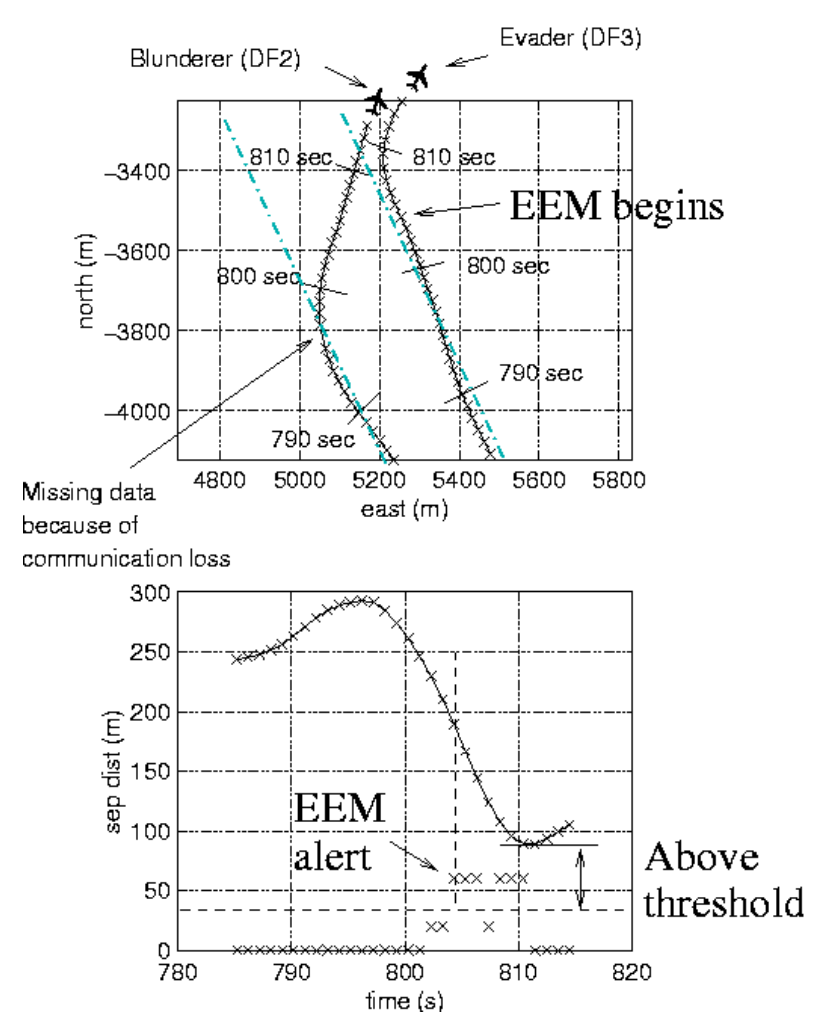

(a)
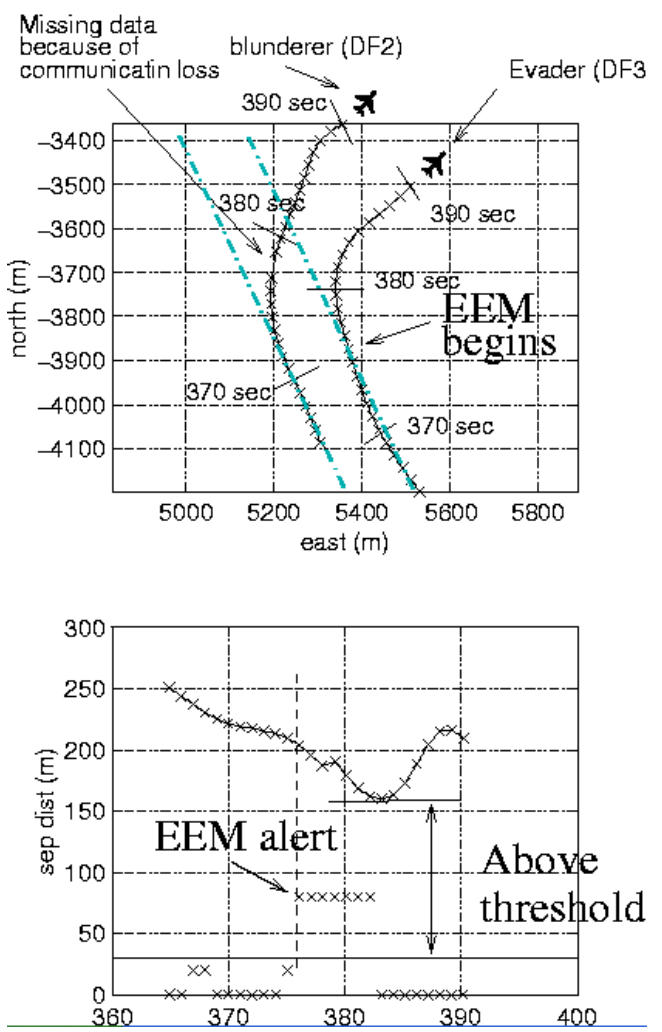

(b)

Figure 12. Flight Demonstration: Independent Approach Scenario. 


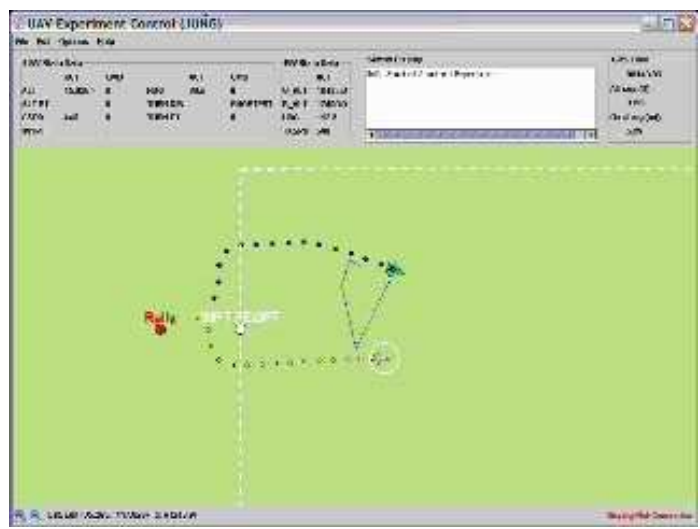

(a) Pursuer blunders into the path of evader and the algorithm computes corresponding danger zone.

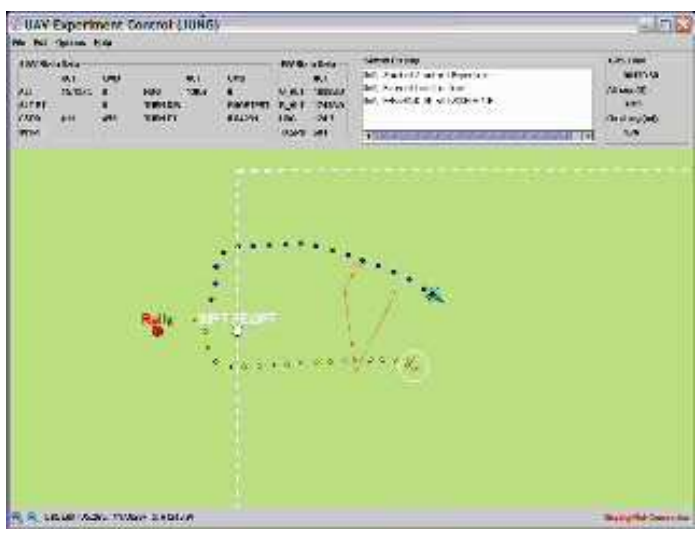

(c) Pursuer touches the danger zone and the algorithm commences an EEM.

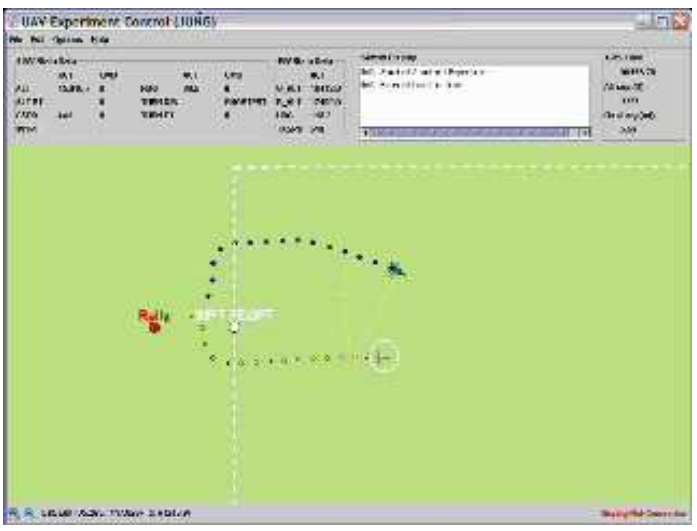

(b) Pursuer enters a caution zone and the pilot is alerted.

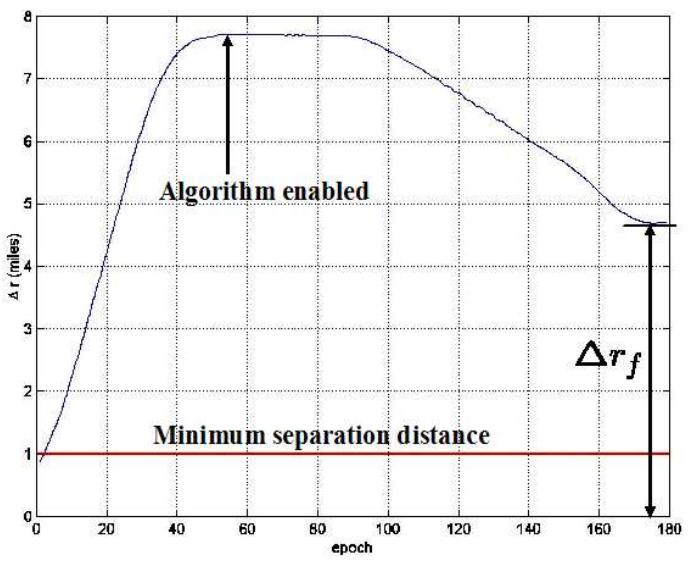

(d) Separation distance between aircraft during the experiment.

Figure 13. Snap shots of the flight experiment.

\section{B. Software Enabled Control CAPSTONE Demonstration using the Boeing Open Control Platform}

The danger zone computation algorithm for Software Enabled Control (SEC) Capstone demonstration sponsored by DARPA and Boeing was also evaluated successfully on the Boeing Open Control Platform (OCP). The experiment involved two aircraft (F-15 and T-33), where one aircraft is assigned as the pursuer (F-15) and the other as the evader (T-33). The flight tests were performed in Edward Airforce Base on June 2004, and the Stanford had six sorties during three flight experiments. The algorithm proved the guaranteed safety (maintaining a minimum separation distance) for every single flight test performed.

Figure 13 shows snapshots of a flight experiment displayed on the Experimental Controller Display installed on both pursuer and evader. As seen in Figure 13(a), as the pursuer blunders into the path of the evader, the algorithm computes the corresponding danger zone based on position and velocity information of two aircraft, yet the evader does not commence an emergence escape maneuver (EEM). As the pursuer enters a caution zone (which is 3,000 ft away from the danger zone) as shown in Figure 13(b), the algorithm alerts the pilot by displaying a warning message as well as changing the color of the danger zone. Finally, once the pursuer touches the danger zone, the algorithm commences an EEM immediately based on which part of the danger zone the pursuer touches. Figure 13(d) shows the resulting separation distance between aircraft during this experiment and it is seen that the chosen minimum separation distance (1 mile radius of circle) was well maintained. 


\section{Conclusions}

The flight demonstration of an optimal control theoretic approach, with some aspects from differential game theory, for computing the danger zones for closely spaced parallel approaches has been presented. This is the first time this new approach has been flight demonstrated to this important problem. If the correct danger zone could be calculated, then it would be possible to get $100 \%$ of clear-day capacity in poor-visibility days even on 750 foot runways. The danger zones vary significantly (during an approach) and calculating them in real time would be very significant. Approximations (e.g. outer bounds) are not good enough. This paper has presented a flight demonstration of calculating these danger zones in real time for a very broad class of blunder trajectories.

Compared to previous algorithms, the new approach in this paper enables a more extensive range of blunder trajectories and variations in the evader emergency evasive maneuver to be considered and to be evaluated definitively. This approach guarantees that safe emergency evasive maneuvers can be conducted if effected outside the danger zone and if the maneuver severity of both the blunderer and evader are within the bounds assumed in computing the danger zone for each case as it comes up.

The implementation issues, which include communication losses and a cockpit display, are addressed, and the algorithm has been validated with over a million simulation runs where the adjacent aircraft performed a comprehensive range of blunder trajectories. The validation shows that the algorithm could successfully alert and prevent collisions in all $100 \%$ of the simulations ran.

A fully autonomous Dual Unmanned Aerial Vehicle test bed was developed. The danger zone computation algorithm has been demonstrated with the test bed in both ground taxiing and flight. The algorithm was proved to be generic and adaptable throughout the evaluation using the Boeing OCP, and the demonstration showed that the algorithm can be successfully implemented and that it can run in real-time.

Finally, with the necessary sensors and datalink, and the appropriate procedures in place, the new CSPA algorithm demonstrated in this paper will enable provably safe closely spaced parallel approaches, in poor visibility, to airports with runways as close as $750 \mathrm{ft}$. This will restore the arrival rates under these conditions to the original levels that are achievable during good visibility.

\section{Acknowledgments}

This research is supported by the DSTA, Singapore, the DARPA Software Enabled Control (SEC) program administered by AFRL under contract F33615-99-C-3014, and by the NASA Joint University Progam.

\section{References}

\footnotetext{
${ }^{1}$ Pritchett, A. R., "Pilot Performance at Collision Avoidance During Closely Spaced Parallel Approaches," Air Traffic Control Quarterly, Vol. 7, No. 1, 1999, pp. $47-75$

${ }^{2}$ Enge, P., Walter, T., Pullen, S., Key, C., Chao, Y. C., and Tsai, Y. J., "Wide Area Augmentation of the Global Positioning System," Proceedings of the IEEE, Vol. 84, No. 8, August 1996, pp. 1063-1088.

${ }^{3}$ Radio Technical Commission for Aeronautics, "Minimum Aviation System Performance Standards for Automatic Dependent Surveillance-Broadcast (ADS-B)," Tech. rep., RTCA, February 1997, RTCA-186 DRAFT 4.0.

${ }^{4}$ Haines, A. L. and Swedish, W. J., "Requirements for independent and dependent parallel instrument approaches at reduced runway spacing," Tech. Rep. FAA-EM-81-8, The MITRE Corporation, May 1981.

${ }^{5}$ Koczo, S., "Coordinated Parallel Runway Approaches," Tech. Rep. NASA Contractor Report 201611, Rockwell International, Cedar Rapids, Iowa, October 1996.

${ }^{6}$ Bone, R., Mundra, A., and Olmos, B. O., "Paired Approach Operational Concept," Proceedings of the Digital Avionics Systems Conference (DASC01), Daytona FL, October 2001.

${ }^{7}$ Hammer, J., "Study of the Geometry of a Dependent Approach Procedure to Closely Spaced Parallel Runways," Proceedings of the IEEE/AIAA 18th Digital Avionics Systems Conference, St. Louis MO, 1999, pp. 4.C.3-1.

${ }^{8}$ Elliot, D. and Perry, B., "NASA Research for Instrument Approaches to Closely Spaced Parallel Runways," Proceedings of the AIAA Guidance, Navigation and Control Conference, Denver CO, August 2000, AIAA 2000-4358.

${ }^{9}$ Waller, M. C. and Scanlon, C. H., "A Simulation Study of Instrument Meteorological Condition Approaches to Dual Parallel Runways Spaced 3400 and 2500 feet Apart Using Flight-Deck-Centered Technology," Tech. Rep. NASA/TM-1999208743, Langley Research Center, Hampton, Virginia, March 1999.

${ }^{10}$ Gazit, R. Y., Aircraft Surveillance and Collision Avoidance using GPS, Ph.D. thesis, Department of Aeronautics and Astronautics, Stanford University, 1996.

${ }^{11}$ Gazit, R. Y. and Powell, J. D., "The effect of GPS-based surveillance on aircraft separation standards," Proceedings of IEEE PLANS, April 1996.
} 
${ }^{12}$ Winder, L. F. and Kuchar, J. K., "Evaluation of Collision Avoidance Maneuvers for Parallel Approach," Journal of Guidance, Control and Dynamics, Vol. 22, No. 6, November-December 1999, pp. 801-807.

${ }^{13}$ Houck, S. and Powell, J. D., "A Parametric Sensitivity Study of Ultra Closely Spaced Parallel Approaches," Proceedings of the Digital Avionics Systems Conference (DASC00), Philadelphia PA, October 2000, pp. 2.D.4-1.

${ }^{14}$ Houck, S. and Powell, J. D., "Assessment of the Probability of a Midair Collision during an Ultra Closely Spaced Parallel Approach," Proceedings of the AIAA Guidance, Navigation and Control Conference, Montreal, Canada, August 2001, AIAA-2001-4205.

${ }^{15}$ Houck, S., Multi Aircraft Dynamics Navigation and Operation, Ph.D. thesis, Department of Aeronautics and Astronautics, Stanford University, 2001.

${ }^{16}$ Houck, S., Barrows, A., Parkinson, B., Enge, P., and Powell, J. D., "Flight testing WAAS for use in closely spaced parallel approaches," Proceedings of the ION-GPS 1999 Meeting, Nashville TN, 1999, pp. 1787-1798.

${ }^{17}$ Jennings, C., Threat Displays for Final Approach, Ph.D. thesis, Department of Aeronautics and Astronautics, Stanford University, 2003.

${ }^{18}$ King, B. T. and Kuchar, J. K., "Evaluation of Collision Alerting System Requirements for Paired Approach," Proceedings of the Digital Avionics Systems Conference (DASC00), Philadelphia PA, October 2000, pp. 2.D.1-1.

${ }^{19}$ Haissig, C., Corwin, B., and Jackson, M., "Designing an Airborne Alerting System for Closely Spaced Parallel Approaches," Proceedings of the AIAA Guidance, Navigation and Control Conference, San Diego CA, August 1999, AIAA-993986.

${ }^{20}$ Jackson, M., Samanant, P., and Haissig, C., "Analysis of Airborne Alerting Algorithms for Closely Spaced Parallel Approaches," Air Traffic Control Quarterly, Vol. 9, No. 4, December 2001.

${ }^{21}$ Carpenter, B. and Kuchar, J., "Probability-Based Collision Alerting Logic for Closely-Spaced Parallel Approach," Proceedings of the AIAA 35th Aerospace Sciences Meeting and Exhibit, Reno NV, January 1997, AIAA 97-0222.

${ }^{22}$ Landry, S. and Pritchett, A. R., "The Safe Zone for Paired Closely Spaced Parallel Approaches: Implications for Procedures and Automation," Proceedings of the Digital Avionics Systems Conference (DASCO0), Philadelphia PA, October 2000, pp. 3.E.3-1.

${ }^{23}$ Pritchett, A. R. and Landry, S., "Operational Concepts for closely spaced parallel approaches," Proceedings of the Digital Avionics Systems Conference (DASC00), St. Louis MO, October 1999, pp. 4.C.2-1.

${ }^{24}$ Jennings, C., Charafeddine, M., Taamallah, S., and Powell, J. D., "Flight Demonstration of 3D Perspective Synthetic Vision and ADS(B) for Closely Spaced Parallel Approaches," Proceedings of the Digital Avionics Systems Conference (DASC02), Irvine CA, October 2002.

${ }^{25}$ Landry, S. and Pritchett, A. R., "Displaying Procedural vs. Real-time information for Paired Approaches," Proceedings of the Digital Avionics Systems Conference (DASC00), Irvine CA, October 2002.

${ }^{26}$ Rossow, V. J., Fong, R. K., Wright, M. S., and Bisbee, L. S., "Vortex wakes of two transports measured in 80 by 120 foot wind tunnel," Journal of Aircraft, Vol. 33, No. 2, March-April 1996, pp. 399-406.

${ }^{27}$ Rossow, V. J., "Wake-Vortex separation distances when flight-path corridors are constrained," Journal of Aircraft, Vol. 33, No. 3, May-June 1996, pp. 539-546.

${ }^{28}$ Rossow, V. J. and Tinling, B. E., "Research on Aircraft/Vortex-wake interactions to determine acceptable level of wake intensity," Journal of Aircraft, Vol. 25, No. 6, 1988, pp. 481-492.

${ }^{29}$ Holforty, W., "Wake Vortex Visualization with Synthetic Vision," Proceedings of the Digital Avionics Systems Conference (DASC01), Daytona FL, October 2001.

${ }^{30}$ Holforty, W., Flight-deck display of neighboring aircraft wake vortices, Ph.D. thesis, Department of Aeronautics and Astronautics, Stanford University, 2003.

${ }^{31}$ Hinton, D. A. and O'Connor, C. J., "Development of a wake vortex spacing system for airport capacity enhancement and delay reduction," Proceedings of the Digital Avionics Systems Conference (DASC00), Philadelphia PA, October 2000, pp. 3.E.6-1.

${ }^{32}$ Perras, G. H. and Dasey, T. J., "A statistical analysis of approach winds at capacity-restricted airports," Proceedings of the Digital Avionics Systems Conference (DASC00), Philadelphia PA, October 2000, pp. 3.E.4-1.

${ }^{33}$ Teo, R., Computing Danger Zones for Provably Safe Closely Spaced Parallel Approaches: Theory and Experiment, Ph.D. thesis, Department of Aeronautics and Astronautics, Stanford University, 2003.

${ }^{34}$ Teo, R. and Tomlin, C. J., "Computing Danger Zones for Provably Safe Closely Spaced Parallel Approaches," In the AIAA Journal of Guidance, Navigation, and Control, Vol. 26, No. 3, May/June 2003, pp. 434-442.

${ }^{35}$ Teo, R. and Tomlin, C. J., "Computing Provably Safe Aircraft to Aircraft Spacing for Closely Spaced Parallel Approaches," Proceedings of the Digital Avionics Systems Conference (DASC00), Philadelphia PA, October 2000, pp. 2.D.2-1.

${ }^{36}$ Teo, R. and Tomlin, C. J., "Provably Safe Evasive Maneuvers against Blunders in Closely Spaced Parallel Approaches," Proceedings of the AIAA Guidance, Navigation and Control Conference, Montreal, Canada, August 2001, AIAA-2001-4293.

${ }^{37}$ Kuchar, J. K., "Methodology for Alerting-System Performance Evaluation," Journal of Guidance, Control and Dynamics, Vol. 19, No. 2, March-April 1996, pp. 438-444.

${ }^{38}$ Shank, E. M. and Hollister, K. M., "A Statistical Risk Assessment Model for the Precision Runway Monitor System," Proceedings of the ATCA Conference, 1992.

${ }^{39}$ Jang, J. S., Nonlinear Control using Discrete-time Dynamic Inversion under Input Saturation: Theory and Experiment on the Stanford Dragonfly UAVs, Ph.D. thesis, Department of Aeronautics and Astronautics, Stanford University, 2003. 